\title{
SEGMENT TRANSFER PROTOCOLS FOR A \\ HOMOGENEOUS COMPUTER NETWORK
}

\author{
ERIC MANNING AND R.W. PEEBLES \\ DEPT OF COMPUTER SCIENEE and \\ COMPUTER COMMUNICATIONS NETWORKS GROUP \\ UNIVERSITY OF WATERLOO
}

\section{INTRODUCTION}

This research is focussed on solving certain problems of distributed processing on a distributed data base, with emphasis on transaction processing. Many data bases exhlbit geographic locality of reference; most of the transactions homing on a given component of the data base origlnate from a particular geographic region. At the same time there is a need to operate the collection of components as a single data base, to provide for occasional transactions which cross regional boundaries, and for managerial querles and information retrieval applications which span the entire data base. There are many examples of this associated with business and industry; credit and inventory records for example. Finally, geographic locality of reference is only one of the reasons for creating loglcally unified but physically distributed data bases. If a data base contains information supplied by several agencies, each may insist as a matter of policy that 'Its' data be held in 'its' hardware located on 'its' premises, quite apart from technical efficiencies which may accrue.

The general-purpose, heterogeneous network as exempl ified hy ARPANET (1) or CYCLADES (2) provides one possible vehicle for the support of a distributed data base. However, the advantage of heterogeneity (multiple CPU architectures) carries the penalty of complex protocol needed to overcome incompatibllities. One would orefer a single architecture for all host CPU's if data is the resource to be shared. Moreover, transaction processing often does not require large, expensive CPUS. Modest CPU Dower sufflces to read short requests, perform the simple calculations associated with data base probes, and format short replies. Gur simulations and queueing analyses indicate that a midicomputer is more than adequate if enough disc store and a high channel data rate to disc are provided. We are therefore led to consider networks of identical midicomputers. The cost of such networks could be relatively 
low: perhaps $\$ 500,000$ for five nodes. It is therefore plausible to assume that they could be marketed and managed as turnkey or package systems. Hence we as suine that we are able to specify host hardware, host software and communications subnetwork, as a single, integrated system. The major goal of our research has been to investigate how far these freedoms can be exploited to yield simple, elegant structures.

The communications subnetwork (or subnet) must cater to bursty traffic and have low cost per port. Packet-switching is one possibility, although the price of current packet switches is similar to the cost of our host. We therefore selected a loop based on the Newhall-Farmer protocol as subnet: It is well-suited to bursty traffic and the port cost is a few thousand dollars.

ARPANET has imposed virtual circuits (the link/socket construct) on a message-with-address or MWA subnet, because of the need to support traditional host operating systems. We have taken the opposite approach and have tried to use MWA switching as far as possible. (Transaction traffic is well-suited to the properties of MiNA switching: however, note the 'comment' in our conclusions). Consequently the host operating system is MWA - a so-called Message-Switched Operating System. It is transaction-driven; nothing happens until a transaction arrives. The transaction generates a directed graph of message fiows which are processed to yleld a response message and side-effects.

Finally, each host runs a copy of the same operating system. Moreover, the network is a turnkey package so that users are not required or permitted to write applications programs. This implies that the network environment is "friendiy"; a close degree of loglcal coupling between hosts is possible as the behaviour of other hosts is predictable. This in turn can be exploited to yield efficiency.

\section{ARCHITECTURE OVERVIEW}

There are exactly two primitive objects. The prinitive data object is the segment and the primitive control object is the task. Prograin work areas, messages, portlons of code and individual records of files are all examples of segments. The entities whlch operate on transactions are examples of tasks; files are also examples of tasks.

For each transaction type handled by the system there will be one or more standard sequences of operations required for processing. Each standard sequence is represented 
by a series of code segments called a software machine (SM). This name was chosen to reflect the fact that as we learn more about this type of processing more of the software will migrate to hardware. A task is an ordered pair <SM, message $>$ and is represented by a Task Queue Element (see Figure 1a). All segments are located via their Segment Table Entries (STE) in the Master Segment Table, Figure $1 \mathrm{~b}$. The TOE then identifies the task by holding its name, prlority and pointers to its software machine STE'S (code panel) and data STE's (data panel), as shown in Figure $1 \mathrm{c}$.

\section{Communications}

The communications nucleus consists of a special task in each host, called the Switch; a processor interposed between each host and the communications subnetwork, called the Communications Device; and the communications subnetwork. The function of the nucleus is to move message segments from a sending task to any other task in the same or any other host. The sending task uses exactly the same protocol for both types of message passing (intra-host and inter-host), thus providing a basis for rendering the physical distribution of the data base transparent to users. The Task Queue and Master Segment Table exist in the virtual space of the Switch task in each host.

The communications subnet is a Newhall Loop (4): a high speed digital line, closed on itself, with repeater ports that regenerate signals and allow subscribers to send and receive data. These loop ports have fixed slze buffers so that subscriber messages must be broken into blocks (loop messages) of size no larger than the buffer length (typically 32 bytes to 256 bytes). Only one port is allowed to send at any given time and a "pass control" signal (permission to send) is handed from port to port around the loop. A port can transinit only one buffer (or less) of text and must then pass control to the next port down the line. Each block of text is preceded by a header that contains: 'who to', 'who from', and 'op. code information. Thus the loop is a message with address system and matches the message-switched operating system archltecture nicely. Each loop port examines the 'who to' fields as they go by and recognizes its own address. Messages for a glven host are copled off the loop by the port.

A processor called the Communications Devlce (CD) is interposed between each host and its loop port. The CD is an intelligent Block Transfer Controller that does most of the work during inter-host message transfer. This relieves the host CPU of the trivial communications "mothering" and is important to the efficiency of inter-host message passing. 


\section{Secondary storage}

Secondary storage is managed by a separate CPU called the Disc Machine or DM. The DM runs a message switched operating system identical to its assoclated host. Multiple DMs per host are feasible and the DM speaks to the loop on the same level of protocol as the host. This implies that transactions between a host and a remote DM proceed without reference to the remote host. DM tasks include the files, whose data segments are records and whose procedure segments are the access method. The disc itself is treated as a linear segmented memory for uniformity and simplified storage management (5).

\section{INTER-PROCESS COMMUNICATION}

An obvious way to make Inter-process Communication efficient is to put as much of it as possible in hardware. To this end a Real Network Address space (RNAS) is defined that enables the CPU at any host to directly reference primary storage and secondary storage at any host in the network. This powerful construct must be regulated to avold chaos and this is done by making RNAS avallable to the Switch but not to applications tasks. Furthermore, a Virtual Vetwork Address Space (VNAS) is defined for the switch that gives each host full control of its own resources. A sending task invokes its local switch by generating an interrupt and passing the following parameters:

send 〈 sendid, rcvid, msgid, Soption, Roption >

where: sendid - name of sending task (as in TQE)

$$
\begin{aligned}
& \text { rcvid - name of receiving task (as in TQE) } \\
& \text { msgid - name of segment to be moved } \\
& \text { Soption - 'destroy' or 'preserve' the sending task } \\
& \text { Roption - 'create' or ' append' the recelving task }
\end{aligned}
$$

The 'create' Roption is used to spawn a new task in the processing of a transaction and results in the creation of a TQE that links the named ( rcvld) software machine to the message segment.

Intra-Host IPC

To pass a message between two tasks executing in the same host we use a method due to $S$. Wecker (3). The Switch simply coples a the segment pointer from the sender's data panel to the recelver's data panel. These are located by searching the Task Queue for 'rcvid' and the sender's data panel for 'msgid'. 
Inter-Host IPC

The sending Switch task requests "perinission to transfer" of the receiving Switch using a VNAS address. The recelving Switch returns an RNAS address pointing to an empty segment nominated to receive the message. The sending CD then fetches the message segment from its hosts' storage, ireaks it into loop messages and transmits them. The receiving is reassembles the segment and stores it in the nominated area; note that host CPUs do not participate in the message transfer after the initial exchange between switches. The $C D$, therefore, serves to interface between the I Inear segmented structure of host tasks and the inessage with address structure of the loop subnet. The detalls of this are explained in (6).

\section{Elow Control}

Flow control occurs at two levels. At the logical, or task level, message transmission can be blocked by the receiving task. All STE's have a field that indicates whether or not they can be used for message reception. If the Switch can find no "receiving" STE's in the recelver's data panel it returns a 'transmission blucked' signal to the sender. A receiver thus regulates its input by refusing to mark any segments for message reception.

At the physical level a host can regulate input from other hosts through its communications Device. The transfer of a block of text between two hosts involves establishing a short "call" between loop ports as illustrated below:

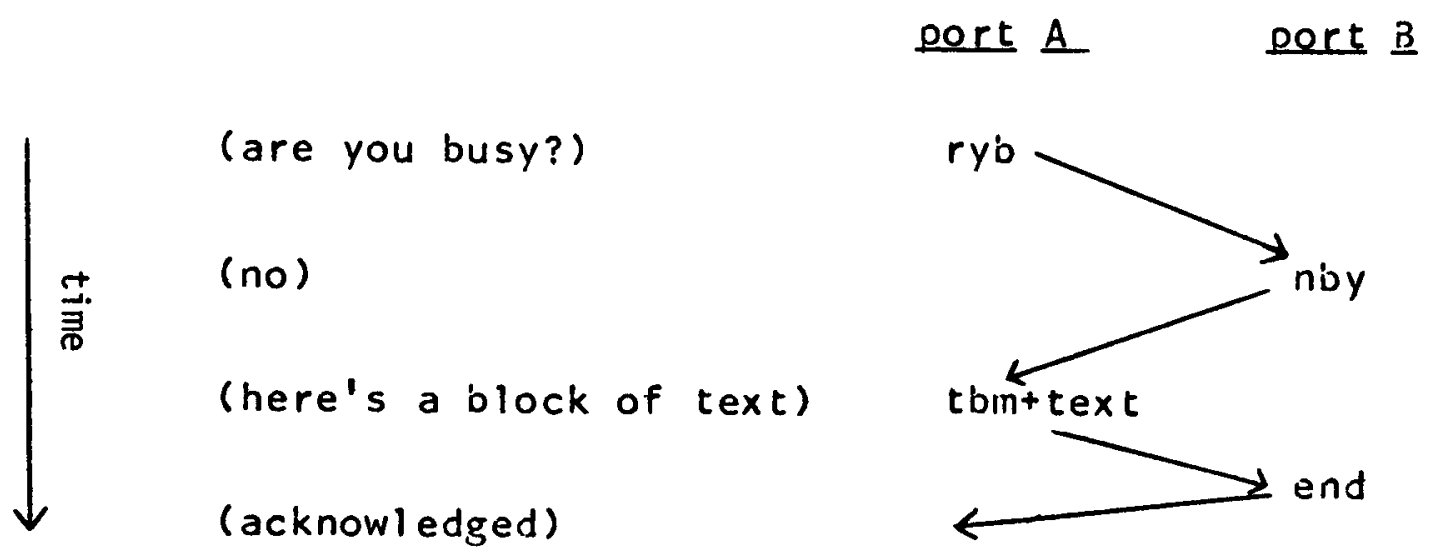

A receiving port wlll not accept traffic if the attached $C D$ or host has no room to store another buffer full of text. It indicates this by returning a ' $B \widehat{S} Y^{\prime}$ signal when a sending port tries to establish a call. When a call is established it is held until the sender's buffer has been transferred. 
If loop buffers are short, thls process of establishing a call represents a significant transinission overhead but it provides a very simple flow control inechanism.

Note that the recelver tears down the call by sending an END and tinis acts as an acknowledgement. Originally it also had the effect of marking the recelver 'not busy' again and thus was witheld until storage for another block of text was avallable. This is clearly undesirable, however, as it allows the receiving host to tie up the sending host. We have therefore adjusted our design to distinguish between the two states: 'busy in a call' and 'out of storage space'.

\section{CONCLUSIOINS}

\section{A Comment on circuit switching}

In the introduction we disavowed circult switcining and stressed the concept of message-switching, but circuitswitching has crept into the design:

- loop ports establish "physlcal" circuits over the shared loop which persist during the transfer of one loop data message.

- Switches establish virtual circults for interhost message passing so that the separately transmitted blocks of a given message can be reassembled.

We feel this is appropriate. Message-switching is used at the transaction level and virtual circuit switching is introduced at the base level to preserve ordering. It should be noted, however, that the circuits are held for only a few milliseconds. Such "fully electronic circult switching" is simply one approach to implementing a message switching facility.

\section{Performance Analysis}

Detailed analytic models and simulations have been used to predict the performance characteristics of the network (7), (8). They strongly indicate that transactions requiring "over the network" servlce can be handled almost as quickly as those serviced locally. Loop speeds in the range of 10 to 20 kilobits per second seem reasonable for networks of 5 to 20 hosts, each supporting 10 to 50 terminals. Clearly these numbers depend heavily upon transaction arrival rates, message length, etc, but the numbers above give an order of magnitude feel for the results we have obtained. 


\section{Implementation}

A two-node prototype network is currently being implemented, using PDP/11 model 45's for the hosts and model 20 's for the CD's and DM's. To date, the CD has been designed and simulated in PL/1, the Switch has been designed and a Newhall Loop is imminently due to arrive at Waterloo. 


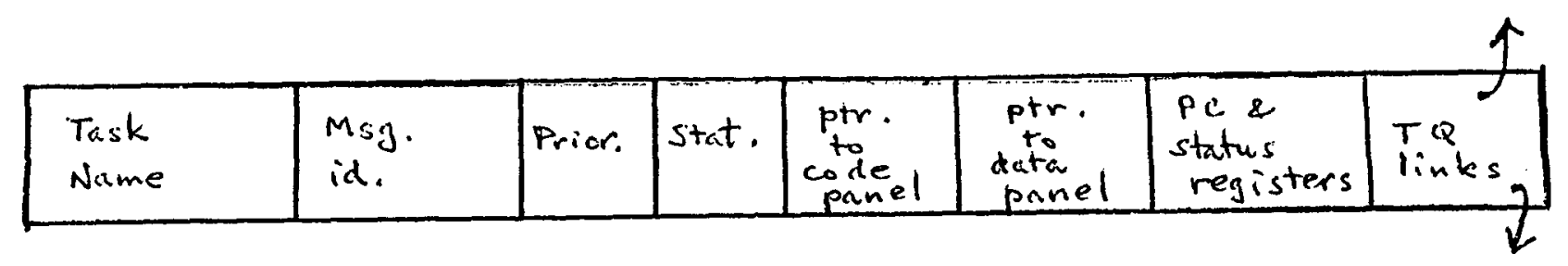

(A) TASK QUEUE ENTRY (TQE)

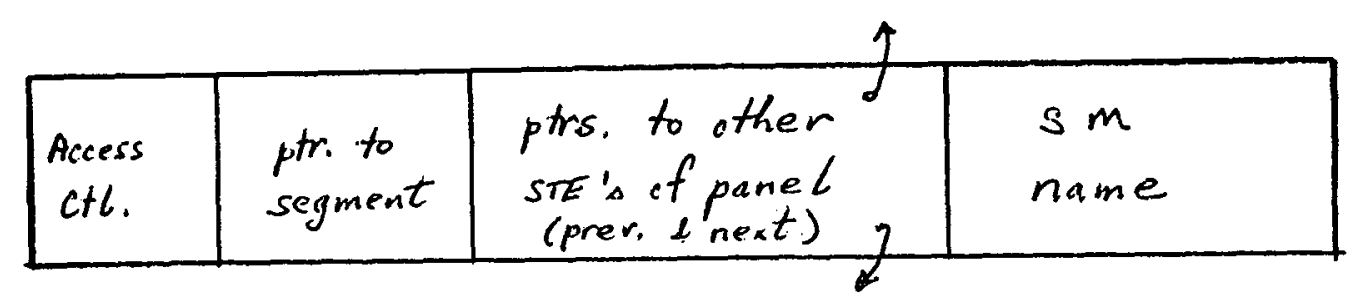

(B) SEGMENT TABLE ENTRY (STE)

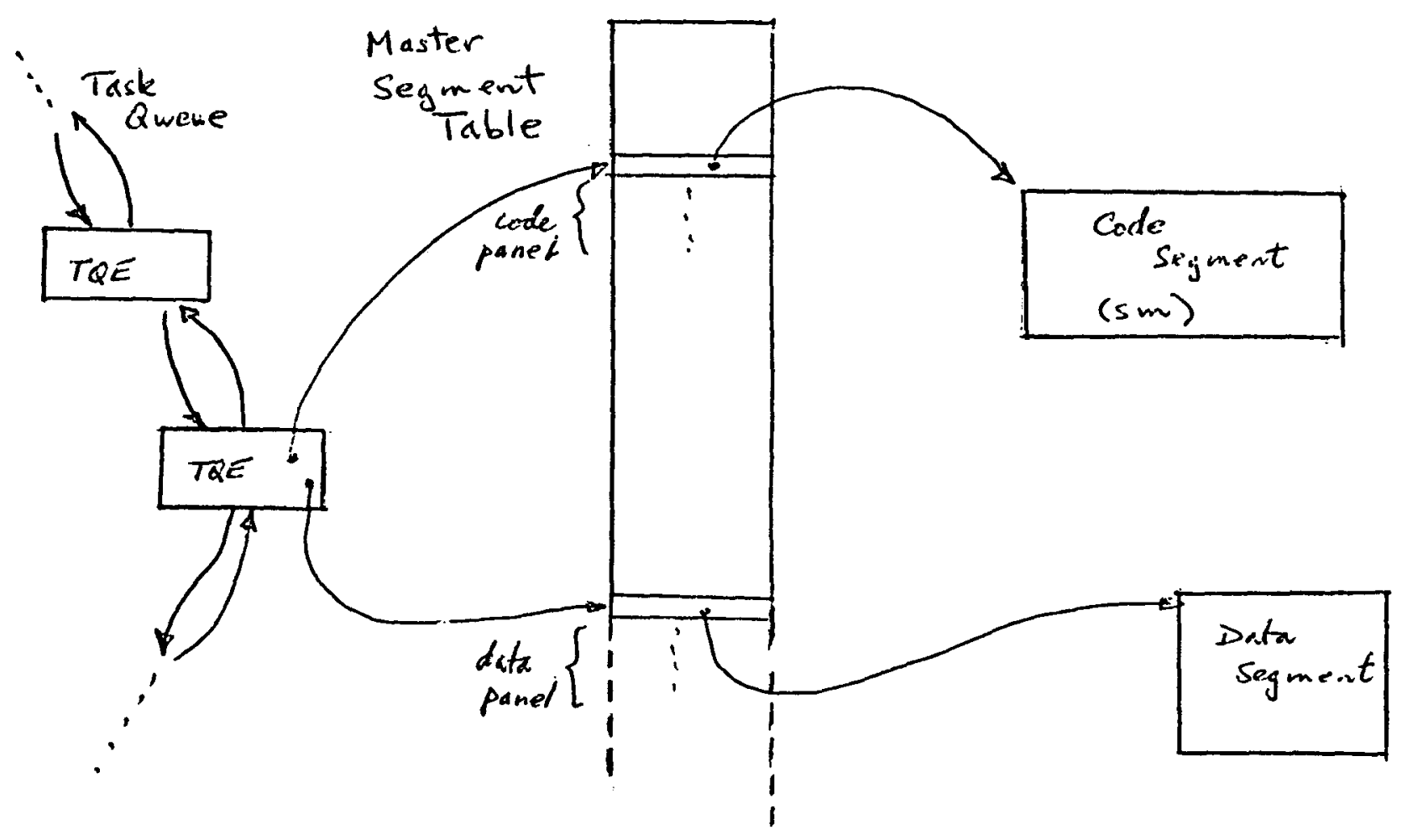

(C) TASK QUEUE AND MASTER SEGMENT TABLE OF THE SWITCH FIGURE 1: CONTROL ELEMENTS IN EACH HOST 


\section{REFERENCES}

(1) Carr, S., Crocker, S. and Cerf, V.; "Host-Host Communication Protocol in the ARPA network", Proceedings of the sJCC-1970, pp. 589-598.

(2) Pouzin, L.: "Presentation and Major Design Aspects of the Cyclades Computer Network", in Data Networks, Analysis and Design, 3rd Data Communications Symposium, (ACM-IEEE, Nov. 13-15, 1973), pp. 80-89.

(3) Wecker, 3.: "A Building Block Approach to MultiFunction Multiple Processor operating systems", Proceedings AIAA Computer Network systems Conference. April 16-18, 1973 .

(4) Newhall, E. E. and Farmer, W. D.; "An Experimental Distributed Switching system to Handle Bursty Conputer Traffic", Proceedings of the ACM Conference, Pine Mountain, Georgia, Jct. 69.

(5) R.W. Peebles and E.G. Manning; "A Homogeneous Network for Data Sharing: Software Arcinitecture and Data Management", CCNG Report E-16, University of Waterloo, Waterloo, Ontario, April, 1974.

(6) E.G. Manning and R. W. Peebles; "A Homogeneous Ne twork For Data Sharing - Comnunications", CCNG Report E-12, University of Waterloo, Waterloo, Ontarlo, March, 1974 .

(7) R.W. Peebles, D. L. Buck and E.G. Manning; "simulation Studies of a Homogeneous Computer Network for Data Sharing", CCNG E-19, University of Waterloo, Waterloo, Ontario, March, 1974 .

(8) Labetoulle, J., Peebles, R.W. and Manning, E.G.; "Performance Analys is of a Homogeneous Computer Network", (Nov. 1974, in preparation). 